\title{
Novel Efficient Classifiers Based on Data Cube
}

\author{
Lixin Fu, University of North Carolina at Greensboro, USA
}

\begin{abstract}
Existing decision tree algorithms need to recursively partition dataset into subsets according to some splitting criteria. For large data sets, this requires multiple passes of original dataset and therefore is often infeasible in many applications. In this article we use statistics trees to compute the data cube and then build a decision tree on top of it. Mining on aggregated data will be much more efficient than directly mining on flat data files or relational databases. Since data cube server is usually a required component in an analytical system for answering OLAP queries, we essentially provide "free" classification. Our new algorithm generates trees of the same prediction accuracy as existing decision tree algorithms such as SPRINT and RainForest, but improves performance significantly. In this article we also give a system architecture that integrates DBMS, OLAP, and data mining seamlessly.
\end{abstract}

Keywords: classification; data cube; decision trees

\section{INTRODUCTION}

Data classification is a process of building a model from available data called training data set and classifying the objects according to their attributes. It is a well-studied important problem (Han \& Kamber, 2001), and has many applications in insurance industry, tax and credit card fraud detection, medical diagnosis, and so forth.

The existing decision tree algorithms need to recursively partition dataset into subsets physically according to some splitting criteria. For large data sets, building a decision tree this way requires multiple passes of the original dataset, therefore, is often infeasible in many applications. In this article we present a new approach of constructing decision trees using a pre-computed data cube.

Our main contributions in this article include:

- designing a new decision tree classifier built on data cube, and

- proposing an architecture that takes the advantages of above new algorithm and integrates DBMS, OLAP systems, and data mining systems seamlessly. 
The remainder of the article is organized as follows. The next section gives a brief summary of the related work. In the third section, statistics tree structures and related data cube computation algorithms are described as the foundation for later sections. An architecture that integrates DBMS, OLAP, and data mining functions is proposed in the fourth section. The fifth section describes our new cube-based decision tree classification algorithm called cubeDT. Evaluation of cubeDT is given in the sixth section. Finally, we summarize the article and discuss the directions of our related future work.

\section{BACKGROUND}

Decision trees have been widely used in data classification. As its precursor algorithm ID-3 (Quilan, 1986), algorithm C4.5 (Quilan, 1993) generates a simple tree in a top-down fashion. Data are partitioned into subsets recursively according to best splitting criteria determined by highest information gain until the partitions contain samples of the same classes. For continuous attribute $\mathrm{A}$, the values are sorted and the midpoint $\mathrm{v}$ between two values is considered as a possible split. The split form is A $\mathrm{d} \leq \mathrm{v}$. For a categorical attribute, if its cardinality is small, all subsets of its domain can be candidate splits; otherwise, we can use a greedy strategy to create candidate splits.

SLIQ (Mehta, Agrawal, \& Rissanen, 1996) and SPRINT (Shafer, Agrawal, \& Mehta, 1996) are more recent decision-tree classifiers that address the scalability issues for large data sets. Both use Gini index as impurity function, presorting (for numerical attributes), and breadth-firstsearch to avoid resorting at each node. Both SLIQ and SPRINT are still multi-pass algorithms for large data sets due to the ne- cessity of external sorting and out-ofmemory structures such as attribute lists.

\section{Surajit, Chaudhuri, Fayyad, and} Bernhardt (1999) give a scalable classifier over a SQL database backend. They develop a middleware that batches query executions and stages data into its memory or local files to improve performance. At its core is a data structure called count table or CC table, a four-column table (attributename, attribute-value, class-value, count). Gehrke et al. give a uniform framework algorithm RainForest based on AVC-group (a data structure similar to CC tables but as independent work) for providing scalable versions of most decision tree classifiers without changing the quality of trees (Gehrke, Ramakrishnan, \& Ganti, 1998). With usually much smaller sizes of CC tables or AVC-group than the original data or attribute lists in SPRINT, these two algorithms generally improve the mining performance. However, they together with all other classification algorithms (as far as we know) including SLIQ and SPRINT still need to physically access (sometimes in multiple scans) original data set to compute the best splits, and partition the data sets in the nodes according to the splitting criteria. Different from these algorithms, our cube-based decision tree construction does not compute and store the F-sets (all the records belonging to an internal node) to find best splits, nor does it partition the data set physically. Instead, we compute the splits through the data cubes, as shown in more detail in the fifth section.

The BOAT algorithm (Gehrke, Ganti, Ramakrishnan, \& Loh, 1999) constructs a decision tree and coarse split criteria from a large sample of original data using a statistical technology called bootstrapping. Other classification methods include Bayesian classification (Cheeseman \& Stutz, 1996), back propagation (Lu, Setiono, \& 
Liu, 1995), association rule mining (Lent, Swami, \& Widom, 1997), k-Nearest neighbor classification (Duda \& Hart, 1973), and so forth. Recently, a statistics-based classifier is built on top of data cube (Fu, 2003).

Since cubeDT is built on top of the technologies of OLAP and data cube, the performance of cube computation has a direct influence on it. Next, we briefly introduce some of the cube systems and cube computation algorithms. To compute data cubes, various ROLAP (relational OLAP) systems, MOLAP (multidimensional OLAP) systems, and HOLAP (hybrid OLAP) systems are proposed (Chaudhuri \& Dayal, 1997). Materialized views and indexing are often used to speedup the evaluation of data cubes and OLAP queries.

Materializing all the aggregate GROUP_BY views may incur excessive storage requirements and maintenance overhead for these views. A view selection algorithm proposed by Harinarayan, Rajaraman, and Ullman (1996) uses a greedy strategy to choose a set of views over the lattice structure under the constraint of certain space or certain number of views to materialize. Agarwal et al. ( 1996) overlap or pipeline the computation of the views so that the cost of the processing tree is minimized. For sparse data, Zhao, Deshpande, and Naughton proposed the chunking method and sparse data structure for sparse chunks (1997).

For dimensions with small cardinalities, bitmap indexing is very effective (O’Neil, 1987). It is suitable for ad-hoc OLAP queries and has good performance due to quick bitwise logical operations. However, it is inefficient for large domains, where encoded bitmap (Chan \& Ioannidis, 1998) or B-trees (Comer, 1979) can be used. Other work related to indexing includes variant indexes (O’Neil \& Quass,
1997), join indexes, and so forth. Beyer and Ramakrishnan (1999) develop BUC (bottom-up cubing) algorithm for cubing the group-bys that are above some threshold. Johnson and Shasha (1997) propose cube trees and cube forests for cubing. In order to improve the performance of ROLAP algorithms, which often require multiple passes for large data sets, a multidimensional data structure called Statistics Tree (ST; Fu \& Hammer, 2000) has been developed. The computation of data cubes that have arbitrary combination of different hierarchy levels is optimized in (Hammer \& Fu, 2001). Other important recent work include Dwarf (Sismanis, Deligiannakis, Roussopoulos, \& Kotidis, 2002) and QC-trees (Lakshmanan, Pei, \& Zhao, 2003).

\section{SPARSE STATISTICS TREES}

An ST tree is a multi-way and balanced tree with each level in the tree (except the leaf level) corresponding to an attribute [24]. Leaf nodes contain the aggregates and are linked to facilitate the storage and retrieval. An internal node has one pointer for each domain value, and an additional "star" pointer representing the entire attribute domain, that is, the special $A L L$ value.

ST trees are static structures. Once the number of dimensions and their cardinalities are given, the shape of the tree is set and will not change while inserting new records. The ST tree has exactly $(\mathrm{V}+1)$ pointers for an internal node, where $\mathrm{V}$ is the cardinality of the attribute corresponding to the level of the node. There is a serious problem of this static ST tree structure: when many dimensions have large cardinalities, the ST tree may not fit into memory, thus incurring too many I/Os for 


\section{Figure 1. SST tree example}

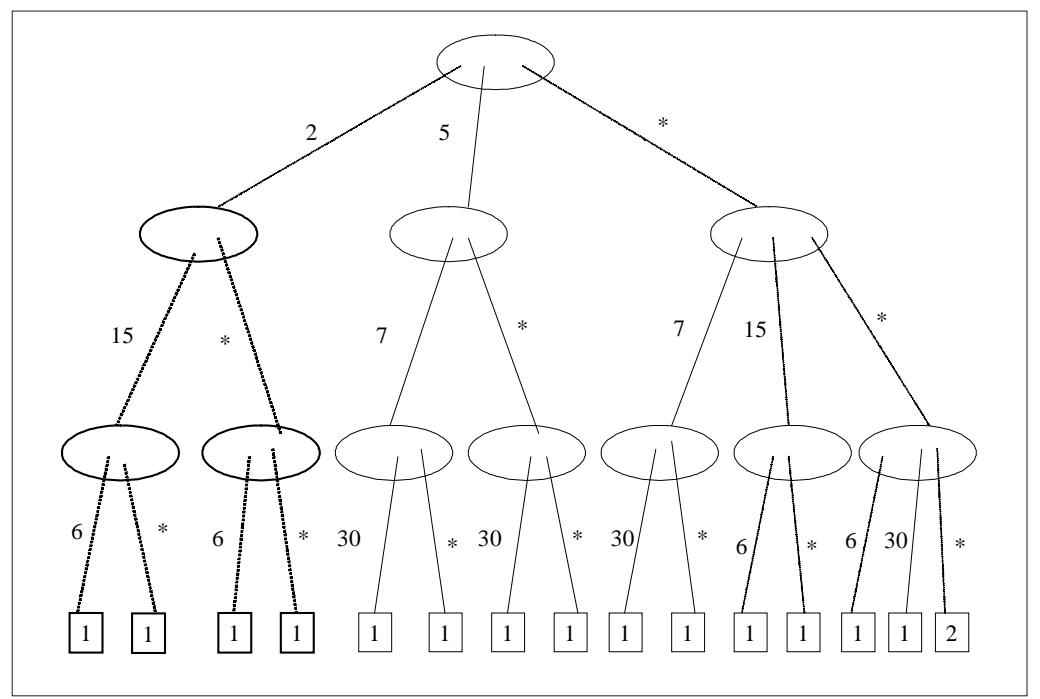

insertions. To address this issue, we develop a new data structure called SST (sparse statistics trees) and related algorithm to evaluate data cubes.

SST is very similar to ST but the pointers are labeled with attribute values instead of implied contiguous values. When a new record is inserted into SST, attribute values are checked along the paths with the existing entries in the nodes. If not matched, new entries will be added into the node and new subtrees are formed. Different from ST trees, where the internal nodes have pointers of contiguous indexes, an SST tree's pointers have labels of corresponding attribute values not necessary contiguous. Figure 1 shows an SST tree after inserting the first two records $(5,7,30)$ and $(2,15,6)$. The paths accessed or newly created while inserting the second record are shown in dashed lines.

If the number of records is large in the training data set, at some point during the insertion process, SST may not fit into memory anymore. A cutting phase is then started, which deletes the sparse leaves and save them on disk for later retrieval. The leaves that are cut in a phase form a run. After all input records have been inserted the runs are merged. The dense cubes are re-inserted into SST but the sparse cubes are stored on disks. While evaluating a cube query after SST initialization, we first check the in-memory SST tree. Starting from the root, one can follow all the pointers corresponding to the constrained attribute values specified in the query for the dimension of that node, to the next level nodes. Recursively descending level by level, eventually we reach the leaves. All the values in the fall-off leaves are summed up as the final answer to the input query. Sparse leaves are retrieved from the merged run stored on disks.

\section{ARCHITECTURE}

Differently from transactional processing systems, for example commercial DBMS, OLAP and data mining are mainly used for analytical purposes at the organi- 
Figure 2. System architecture that integrates DBMS, OLAP, and OLAM

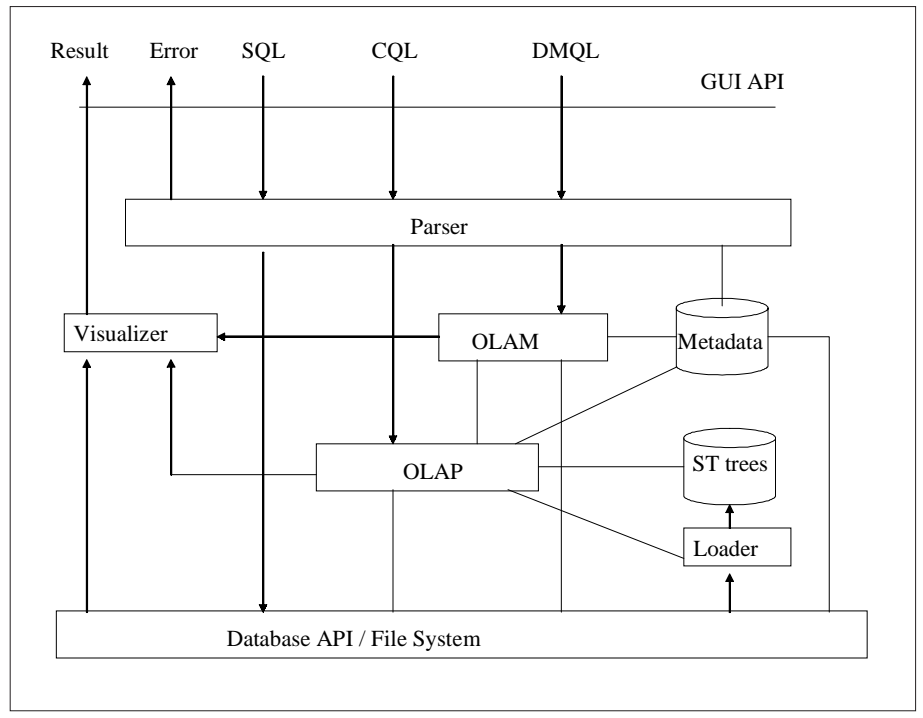

zational level. "A data warehouse is a subject-oriented, integrated, time-variant, and nonvolatile collection of data in support of management's decision making process" (Inmon, 1996).

There are some advantages of deploying data analysis on top of data warehouses. First, data is clean and consistent across the whole organization. Secondly, we can also use the existing infrastructure to manipulate and manage large amounts of data. Thirdly, the DBMS over a data warehouse can choose any interested subset of data to mine on, implementing an adhoc mining flexibility. OLAP and data mining algorithms can give "big picture" information and interesting patterns. OLAM (online analytical mining) system integrates OLAP with data mining and mining knowledge in multidimensional databases. A transaction-oriented commercial DBMS alone is, however, not up to efficient evaluation of complex ad-hoc OLAP queries and effective data mining because DBMS has different workloads and requirements. A natural solution is then to integrate three systems tightly. Figure 2 is our proposed architecture for such an integrated system.

The undirected lines represent bi-directional information flows. Users can submit SQL, CQL, and DMQL (data mining query language) queries through a common GUI API interface. The parser parses the user inputs and dispatches to the corresponding DBMS, OLAP, and OLAM engines if no syntactic errors are detected. Otherwise, the error messages are returned. Related metadata information is stored and will be used later by the data processing engines. The running results from the engines can be represented in various formats, such as diagrams, tables, and so forth, through a visualizer.

In addition to mining directly on databases or files, the OLAM engine can also be built on top of OLAP engines, which is the main topic of this article. The OLAP, or data cube server, instructs a loader to construct ST trees from databases or files so that later on the cube queries are evalu- 
ated using the initialized ST trees (or SST trees), which is significantly faster than using DBMS servers (Hammer \& Fu, 2001). After the ST tree is initialized, the data cubes can be extracted from the leaves to construct decision trees.

\section{CONSTRUCTION OF DECISION TREES USING DATA CUBE}

\section{A General Template of Building Decision Trees}

In decision tree classification, one recursively partitions the training data set until the records in the sub-partitions are entirely or mostly from the same class. When the data cubes have been computed, in this section we will design a new decision tree algorithm which builds a tree from data cubes without accessing original training records anymore.

The internal nodes in a decision tree are called splits, predicates to specify how to partition the records. The leaves contain class labels into which the records satisfying the predicates along the root-to-leaf paths are classified. We consider binary decision trees though multi-way trees are also possible. The following is a general template for almost all decision tree classification algorithms:

\section{Partition (Dataset S) \{}

If (all records in $\mathrm{S}$ are of the same class) then return;

Compute the splits for each attribute;

Choose the best split to partition $\mathrm{S}$ into

$$
\mathrm{S}_{1} \text { and } \mathrm{S}_{2} \text {; }
$$

Partition $\left(\mathrm{S}_{1}\right)$;

\}

Partition $\left(\mathrm{S}_{2}\right)$;

An initial call of Partition (training dataset) will setup a binary decision tree for the training data set. Before the evaluation of the splits, the domain values of the training records are all converted into integers starting from 0 . The conversions can be done during the scanning of original training data.

\section{Compute the Best Split for the Root}

Given a training dataset with $\mathrm{N}$ records each of which has d predictor attributes and the classifying attribute B, suppose that they are classified into $C$ known classes $\mathrm{L}_{\mathrm{p}}, \mathrm{p}=0,1, \ldots, \mathrm{C}$ - 1 . We use giniindex to compute the splits at the root of the decision tree as follows.

$\operatorname{gini}(S)=1-\sum_{j=0}^{C-1} p_{j}^{2}$,

where $p_{j}$ is the frequency of class $j$ in $S$ $p_{j}=\operatorname{count}(B=j) / n, n=|S|$

$\operatorname{gini}(S)=\frac{n_{1}}{n} \operatorname{gini}\left(S_{1}\right)+\frac{n_{2}}{n} \operatorname{gini}\left(S_{2}\right)$,

if $S$ is partitioned into $S_{1}$ and $S_{2}$,

$n_{1}=\left|S_{1}\right|, n_{2}=\left|S_{2}\right|$

A split for continuous attribute $A$ is of form value(A) $d \leq v$, where $v$ is the upper bound of some interval of index $\mathrm{k}(\mathrm{k}=0$, $1, \ldots, \mathrm{V}-1$, where $\mathrm{V}$ is the total number of values for A). To simplify, let us just denote this as $\mathrm{A} \mathrm{d} \leq \mathrm{k}$.

The following algorithm evaluates the best split for attribute A.

1. $\mathrm{x}[\mathrm{j}]=0$, for $\mathrm{j}=0,1, \ldots, \mathrm{C}-1$; CountSum $=0$;

2. $\operatorname{minGini}=1 ; \operatorname{minSplit}=0$;

3. for $\mathrm{i}=0$ to $\mathrm{V}-1$ do

4. $\quad$ countSum $\leftarrow$ countSum $+\operatorname{count}(A=\mathrm{i})$;

5. $\mathrm{n}_{1}=$ countSum; $\mathrm{n}_{2}=\mathrm{n}$-countSum;

6. squaredSumL, squaredSumH $=0$;

7. for $\mathrm{j}=0$ to $\mathrm{C}-1$ do 
8.

$$
\begin{aligned}
& \text { 8. } \\
& x[j]=x[j]+\operatorname{count}(A=i ; B=j) ; \\
& \text { 9. } \\
& \text { 10. }
\end{aligned}
$$

10.

\section{1. endfor}

12. $\operatorname{gini}\left(\mathrm{S}_{1}\right)=1$-sqSumL;gini $\left(\mathrm{S}_{2}\right)=1-$ sqSumH;

13. $\operatorname{gini}(\mathrm{S})=\mathrm{n}_{1} \operatorname{gini}\left(\mathrm{S}_{1}\right) / \mathrm{n}+\mathrm{n}_{2} \operatorname{gini}\left(\mathrm{S}_{2}\right) / \mathrm{n}$;

14. if $\operatorname{gini}(\mathrm{S})<\operatorname{minGinithen}$

15.

MiniGini=gini(S);minSplit=i;

\section{6. endif}

\section{7. endfor}

Lines 1 and 2 initialize temporary variables countSum and array $\mathrm{x}$, and current minimal gini idex minGini and its split position miniSplit. Lines 3 through 17 evaluate all possible splits $\mathrm{A} d \leq \mathrm{i}(\mathrm{i}=0,1, \ldots, \mathrm{V}-1)$ and choose the best one. Each split partitions data set $\mathrm{S}$ into two subset $\mathrm{S}_{1}=\{\mathrm{r}$ in $\mathrm{S}$ $\mathrm{r}[\mathrm{A}] \mathrm{d} \leq \mathrm{i}\}$ and $\mathrm{S}_{2}=\mathrm{S}-\mathrm{S}_{1}$. Line 4 tries to simplify the computation of the size of $S_{1}$, that is, count $(\mathrm{A} \mathrm{d} \leq \mathrm{i})$ by prefix-sum computation. Similarly, array $x[j]$ is used to compute count $(A d \leq i ; B=j)$ for each class $j(j$ $=0,1, \ldots, \mathrm{C}-1)$ in lines 1 and 8 . All these count expressions are cube queries evaluated by the method in the third section.

For categorical attributes, the splits are of form value $(A) \in T$, where $T$ is a subset of all the attribute values of A. Any such subset is a candidate split.

$\mathrm{n}_{1}=\operatorname{count}(\operatorname{value}(\mathrm{A}) \in \mathrm{T})$, and $\mathrm{n}_{2}=\mathrm{n}-\mathrm{n}_{1}$ $\mathrm{p}_{\mathrm{j}}=\operatorname{count}(\operatorname{value}(A) \in \mathrm{T} ; \mathrm{B}=\mathrm{j}) / \mathrm{n}_{1}$

Knowing how to compute these variables, we can similarly compute the gini(S) for each split and choose the best one, as we did for continuous attributes. The final split for the root is then the split with the smallest gini index among all the best splits of the attributes.

\section{Partitioning and Computing Splits for Other Internal Nodes}

The best split computed above is stored in the root. All existing decision tree algorithms at this point partition the data set into subsets according to the predicates of the split. In contrast, cubeDT does not move data around. Instead, it just virtually partitions data by simply passing down the split predicates to its children without touching or querying the original data records any more at this phase. The removal of the expensive process of data partitioning greatly improves the classification performance.

The computation of splits for an internal node other than the root is similar to the method in the fifth section except that the split predicates along the path from the node to the root are concatenated as part of constraints in the cube query. For example, suppose a table containing customer information has three predictor attributes: age, income, and credit-report (values are poor, good, and excellent). The records are classified into two classes: buy or not buy computer. Suppose the best split of A turns out to be "age $d \leq 30$," and now we are computing splits for the attribute income at node $B$. Notice that value $n_{1}=$ count (income $\mathrm{d} \leq \mathrm{v}$; age $\mathrm{d} \leq 30$ ), and $\mathrm{n}_{2}=$ count(age $d \leq 30)-n_{1}$. Here, we do not actually partition data set by applying the predicate "age $d \leq 30$," instead, we just form new cube queries to compute the splits for the node B. As before, these cube queries are evaluated through partial traversal of ST or SST trees.

At node $\mathrm{C}, \mathrm{n}_{1}=$ count (income $\mathrm{d} \leq \mathrm{v}$; age $>30)$, and $n_{2}=\operatorname{count}($ age $>30)-n_{1}$. All other variables are computed similarly for evaluating the splits. Suppose that after computation and comparison the best split at $\mathrm{B}$ is "income $\mathrm{d} \leq \$ 40,000$." The dia- 


\section{Figure 3. Example of computing splits of non-root internal nodes}

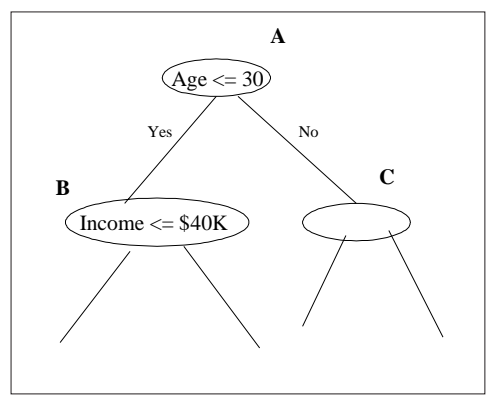

gram shown in Figure 3 gives the initial steps of evaluating splits of nodes A, B, and $\mathrm{C}$.

\section{SIMULATIONS}

To verify the effectiveness of cubeDT, we have conducted preliminary studies by comparing it with bottom-up cubing (BUC; Beyer \& Ramakrishnan, 1999) since the predominant time of cubeDT is spent on the construction of the SST. We compare with BUC because a family of algorithms such as BUC, BUC-BST, Condensed Cube, and QC-trees are all based on recursive partitioning and thus have similar I/O efficiency. In addition, BUC is more than four times faster than MemoryCube (Ross \& Srivastava, 1997), which has shown been superior to previous sparse cube evaluation algorithms. All the experiments are done on a Dell PC Precision 330, which has a $1.7 \mathrm{GHZ} \mathrm{CPU}, 256 \mathrm{MB}$ memory, and the Windows 2000 operating system. All the algorithms are implemented in $\mathrm{C}++$. We used uniformly distributed random data and set each of the five dimensions with a cardinality of 10 . The number of records is increased from 50,000 to 1,000,000 (data set sizes from 1 megabytes to 20 megabytes). The runtimes are shown in Figure 4. SST is about two-four times faster than BUC. The performance im- provements we achieve increase quickly with an increase in the number of records. Note that the runtimes are the times for computing the data cubes.

We also investigate the behavior of SST and BUC by varying the number of dimensions and using data of Zipf distribution (factor is 2). We set the number of records to 100,000 and the cardinality of each dimension is fixed to 20. The number of dimensions increases from 4 to 8 . Figure 5 shows the construction times. Clearly SST is scalable with respect to the number of dimensions.

The query evaluation times are much faster than construction times. We measure the query times using total response times of 100 random queries. The queries are generated by first randomly choosing three dimensions where random numbers within the domains are selected as queried values. All other coordinates in the queries are star values. Since our SST can fit into memories in these experiments, queries can be evaluated without I/Os. SST is one order faster than other BUC (see Figure 6).

These experiments show that SST has a better performance, however, notice that cubeDT is general, that is, the method of computing data cube is not restricted to our cubing algorithms using ST or SST trees. It can be on top of other data cube 
Figure 4. Varying number of records

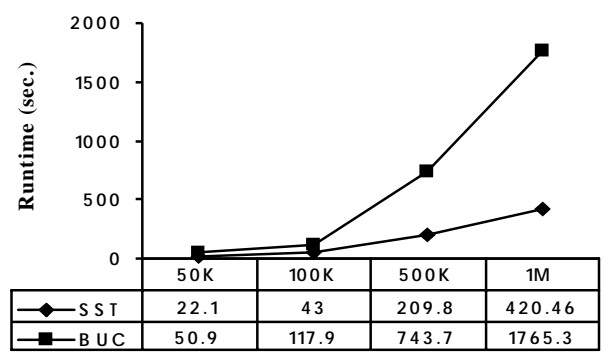

Number of Records

Figure 5. Varying number of dimensions

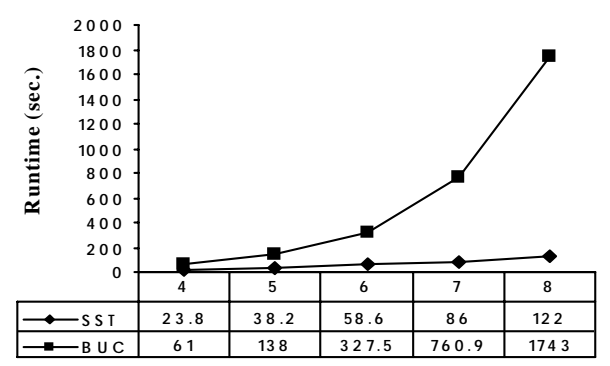

Number of dimensions

Figure 6. Query response times

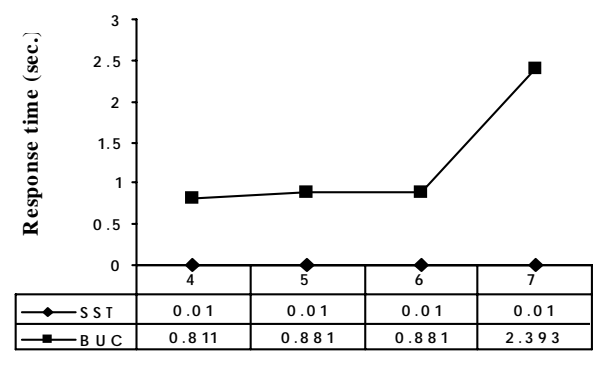

Number of dimensions

systems such as BUC as well. BUC is mainly designed to answer iceberg queries where the cubes whose aggregate values are below certain threshold are pruned. SST trees can also prune those cubes whose aggregate values are below the same threshold. This is easily done by cutting the leaves in SST as shown in the third section. Interested readers may also refer to (Fu, 2004) for more details.
We also compare the performance of cubeDT, which includes both cube computation and decision tree generation phases, with that of RainForest algorithm. According to Mehta et al. (1996) and Shafer et al. (1996), SLIQ produces accurate trees significantly smaller than the trees produced by IND-C4 (a predecessor of C4.5) but is almost one order faster than IND-Cart. SPRINT is faster and more scalable than 
Figure 7. Varying number of records for uniformly distributed data

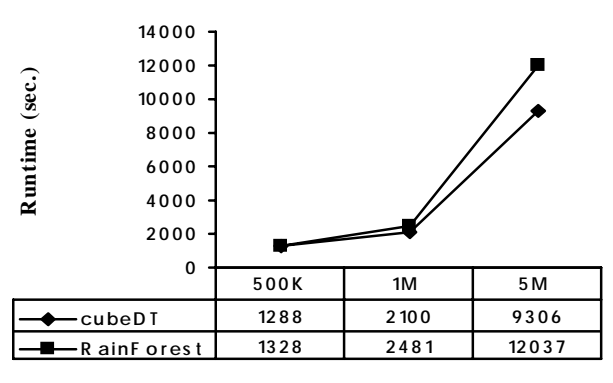

Number of Records

Figure 8. Varying number of records for Zipf distributed data

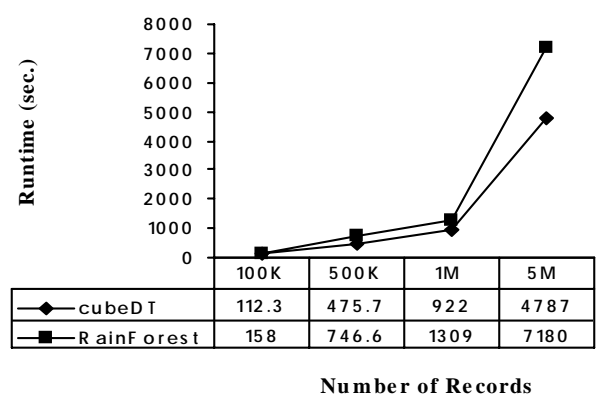

SLIQ while producing exactly the same trees as SLIQ. Previous experiments have also shown that RainForest in Gehrke et al. (1998) offers a performance improvement of a factor five over the previous fastest algorithm SPRINT. So, we compare our cubeDT algorithm with RainForest.

Among several implementations of RainForest such as RF-Read, RF-Write, RF-Hybrid, and RF-Vertical, RF-Read is fastest, assuming that the AVC-groups of all the nodes at one level of the decision tree can fit into memory. In this case, one can only need one scan of reading the input data to compute the AVC-groups at that level and compute the best splits from the AVC-groups. Even in this ideal case (hardly usable in real applications), RainForest needs at least $h$ passes of original potentially large input data set, where $h$ is the height of the decision tree. Other implementations need more read/write passes. In contrast, cubeDT requires one pass of input set to compute the cubes, after that, the decision tree can be built from the data cube without touching the input data any more. In this set of experiments, we use uniform data set containing four descriptive attributes, each of size 10, with the class attribute having five class values. We increase the number of records from half million to five million. Figure 7 shows that cubeDT is faster, and more importantly, the performance gap becomes significantly wider when I/O times become dominant. The runtimes of cubeDT have already included the cube generation times, without which the decision tree construction using cube will be one order faster than RainForest. Similar results are shown in Figure 8 where we use data sets of Zipf 
distribution instead. We use four dimensions whose cardinalities are 10,10,10, and 5, respectively.

Since cubeDT uses the same formulas for computing the splits, it produces the same trees as SLIQ, SPRINT, and RainForest algorithms, that is, they have the same accuracy of classification. The accuracy issue is orthogonal to the performance issue here. However, cubeDT is significantly faster due to direct computation of splits from data cube without actually partitioning and storing the F-sets, especially when input data sets are so large that the I/O operations become the bottleneck of performance.

\section{CONCLUSION AND FUTURE WORK}

In summary, in this article we propose a new classifier that extracts some of the computed data cubes to set-up decision trees for classification. Once the data cubes are computed by scanning the original data once and stored in statistics trees, they are ready to answer OLAP queries. The new classifiers provide additional "free" classification that may interest users. Through the combination of technologies from data cubing and classification based on decision trees, we pave the way of integrating data mining systems and data cube systems seamlessly. An architecture design of such an integrated system has been proposed. We will continue the research on the design of other efficient data mining algorithms on data cube in the future.

\section{REFERENCES}

Agarwal, S., Agrawal, R., Deshpande, P., Naughton, J., Sarawagi, S., \& Ramakrishnan, R. (1996). On the computation of multidimensional aggregates.
In Proceedings of the International Conference on Very Large Databases, Mumbai (Bombay), India, (pp. 506-521). Beyer, K., \& Ramakrishnan, R. (1999). Bottom-up computation of sparse and iceberg CUBEs. In C. Faloutsos (Ed.), Proceedings of the 1999 ACM SIGMOD International Conference on Management of Data (SIGMOD '99), (pp. 359-370), Philadelphia, PA.

Chan, C.Y., \& Ioannidis, Y.E. (1998). Bitmap index design and evaluation. In Proceedings of the 1998 ACM SIGMOD International Conference on Management of Data (SIGMOD '98), Seattle, Washington, (pp. 355-366). Chaudhuri, S., \& Dayal, U. (1997). An overview of data warehousing and OLAP technology. SIGMOD Record, 26(1), 65-74.

Chaudhuri, S., Fayyad, U., \& Bernhardt, J. (1999). Scalable classification over SQL databases. In 15th International Conference on Data Engineering, Sydney, Australia, March 23-26, (p. 470).

Cheeseman, P., \& Stutz, J. (1996). Bayesian classification (AutoClass): Theory and results. In R. Uthurusamy (Ed.), $A d$ vances in Knowledge Discovery and Data Mining, (pp. 153-180). AAAI/ MIT Press.

Comer, D. (1979). The ubiquitous Btree. ACM Computing Surveys, 11(2), 121137.

Duda, R., \& Hart, P. (1973). Pattern classification and scene analysis. New York: John Wiley \& Sons.

$\mathrm{Fu}$, L. (2003). Classification for Free. In International Conference on Internet Computing 2003 (IC'03), Monte Carlo Resort, Las Vegas, Nevada, June 2326.

Fu, L. (2004). Efficient evaluation of sparse data cubes. In Advances in Web-Age Information Management: Fifth Inter- 
national Conference (WAIM'04), Dalian, China, July 15-17, (pp. 336-345). Berlin: Springer-Verlag.

Fu, L., \& Hammer, J. (2000). CUBIST: A new algorithm for improving the performance of ad-hoc OLAP queries. In ACM Third International Workshop on Data Warehousing and OLAP, Washington, D.C., November, (pp. 7279).

Gehrke, J., Ganti, V., Ramakrishnan, R., \& Loh, W.-Y. (1999). BOAT: Optimistic decision tree construction. In Proceedings of the 1999 International Conference on Management of Data (SIGMOD ’99), Philadelphia, PA, June. (pp. 169-180).

Gehrke, J., Ramakrishnan, R., \& Ganti, V. (1998). RainForest: A framework for fast decision tree construction of large datasets. In Proceedings of the 24th VLDB Conference (VLDB '98), New York. (pp. 416-427).

Hammer, J., \& Fu, L. (2001). Improving the performance of OLAP queries using families of statistics trees. In Third International Conference on Data Warehousing and Knowledge Discovery (DaWaK 01), Munich, Germany, (pp. 274-283).

Han, J., \& Kamber, M. (2001). Data mining: Concepts and techniques. Morgan Kaufman Publishers.

Harinarayan, V., Rajaraman, A., \& Ullman, J.D. (1996). Implementing data cubes efficiently. SIGMOD Record, 25(2), 205-216.

Inmon, W. H. (1996). Building the Data Warehouse. New York: John Wiley \& Sons.

Johnson, T., \& Shasha, D. (1997). Some approaches to index design for cube forests. Bulletin of the Technical Committee on Data Engineering, IEEE Computer Society, 20(1), 27-35.
Lakshmanan, L.V.S., Pei, J., \& Zhao, Y. (2003). QC-Trees: An efficient summary structure for semantic OLAP. In A. Doan (Ed.), Proceedings of the 2003 ACM SIGMOD International Conference on Management of Data, San Diego, CA, June 9-12, (pp. 64-75). ACM.

Lent, B., Swami, A., \& Widom, J. (1997). Clustering association rules. In Proceedings of the Thirteenth International Conference on Database Engineering (ICDE '97), Birmingham, U.K., (pp. 220-231).

Lu, H., Setiono, R., \& Liu, H. (1995). NeuroRule: A connectionist approach to data mining. In S. Nishio (Ed.), VLDB'95, Proceedings of 21st International Conference on Very Large Data Bases, Zurich, Switzerland, September 11-15, (pp. 478-489). Morgan Kaufmann.

Mehta, M., Agrawal, R., \& Rissanen, J. (1996). SLIQ: A fast scalable classifier for data mining. In G. Gardarin (Ed.), Advances in Database Technology (EDBT'96), Proceedings of the Fifth International Conference on Extending Database Technology, Avignon, France, March 25-29, (Vol. 1057, pp. 1832). Berlin: Springer-Verlag.

O’Neil, P. (1987). Model 204 architecture and performance. In Proceedings of the Second International Workshop on High Performance Transaction Systems, Asilomar, CA, (pp. 40-59).

O’Neil, P., \& Quass, D. (1997). Improved query performance with variant indexes. SIGMOD Record (ACM Special Interest Group on Management of Data), 26(2), 38-49.

Quilan, J.R. (1986). Introduction of decision trees. In Machine Learning, (Vol. 1, pp. 81-106).

Quilan, J.R. (1993). C4.5: Programs for 
machine learning. Morgan Kaufmann. Ross, K.A., \& Srivastava, D. (1997). Fast computation of sparse datacubes. In Proceedings of the 23rd VLDB Conference (VLDB '97), Athens, Greece, (pp. 116-125).

Shafer, J., Agrawal, R., \& Mehta, M. (1996). SPRINT: A scalable parallel classifier for data mining. In N.L. Sarda (Ed.), VLDB'96, Proceedings of 22nd International Conference on Very Large Data Bases, Mumbai (Bombay), India, September 3-6, (pp. 544-555). Morgan Kaufmann.
Sismanis, Y., Deligiannakis, A., Roussopoulos, N., \& Kotidis, Y. (2002). Dwarf: Shrinking the PetaCube. In Proceedings of the 2002 ACM SIGMOD International Conference on Management of Data (SIGMOD '02), Madison, Wisconsin, USA, (pp. 464 475).

Zhao, Y., Deshpande, P.M., \& Naughton, J.F. (1997). An array-based algorithm for simultaneous multidimensional aggregates. SIGMOD Record, 26(2), 159170.

Lixin Fu is currently an assistant professor at the University of North Carolina at Greensboro (UNCG). He obtained his PhD in computer and information science at the University of Florida in 2001, and his MS in electrical engineering at Georgia Institute of Technology in 1997. Dr. $F u$ joined UNCG in year 2001. His research interests are data mining, data warehousing, databases, and algorithms. He is a co-PI of a half-million ITR NSF grant, and a reviewer for Information Sciences Journal. He is a program committee member of the Twelfth International Conference on Information and Knowledge Management (CIKM 2003), a Carolina Database Research Group member, and member of ACM, IEEE, and Computer Society. 